\title{
TAILPIECE
}

\section{The Evolution Of Casualty Evacuation In The 20th Century (Part 5) - Into The Future}

\author{
MCM Bricknell
}

\begin{abstract}
This is the final part of a series of papers that review the evolution of the military casualty evacuation system in the 20th Century. This paper draws together the themes that have been presented in the previous papers to describe the mandatory functions of such a system. The forward surgical hospital is the key treatment node if wounded soldiers are to have the maximum chance of survival. Suggestions are made for the minimum size, organisation and clinical capability of such a military medical unit. However, the majority of military casualties are likely to be less seriously injured or non-trauma cases. The casualty evacuation system must also have sufficient capacity to accommodate these patients.
\end{abstract}

\section{Introduction}

This is the final part of a series of papers that review the evolution of the military casualty evacuation system in the 20th Century. The first three papers described the system in the British Army. Part 1 describes how, at the turn of the Century, the newly formed Royal Army Medical Corps faced its first test in the Boer War. This experience led to the formation of the Field Ambulance for the collection of casualties from front line units, their initial treatment at the Dressing Station and the transport of casualties to the Clearing Hospital. The large numbers of casualties and the stable front lines of the Western Front of the World War 1 led to an increase in the size and capability of the Casualty Clearing Station (superseding the Clearing Hospital). The care of war wounded having been transformed by the concept of forward surgery. Part 2 discusses how the WW1 organisation of the Casualty Clearing Station was found to be too large and immobile for World War 2. It was reorganised, and additional units created (the Field Surgical Unit, Field Transfusion Unit and the Field Dressing Station) to provide the medical planner with a range of options for the delivery of forward surgery to battle casualties. In Part 3, we see how the Cold War fixed British military medical planning to the challenge faced by huge numbers of surgical and nuclear casualties. This led to a casualty evacuation plan based on large fixed hospital facilities. However, actual military operations in Suez, the Falklands Islands, the Gulf, Former Yugoslavia and Africa, showed that an easily deployable, clinically capable forward hospital facility was a critical component of a military medical system. Part 4 provided an overview of the field medical service in the US Army. This shows how a mobile hospital unit evolved from the French auto chirgue of WW1 into Hospital Platoons in WW2 to the Mobile Army Surgical Hospital and the Forward Surgical Team of the present day. The Israeli Army, the Russian Army and the Croatian Army evolved similar units to provide forward surgical support to military campaigns in the late 20th Century.

This paper concludes this review of military casualty evacuation with a personal view of the themes common to all military medical systems. The organisation and clinical capability of the forward surgical hospital will be discussed in some detail as this unit is the pivot upon which the whole system depends - exactly like the Clearing Hospital of 1910 (1).

\section{THE CASUALTY EVACUATION CHAIN}

UK military clinical doctrine places the 1-24 hour rule as the central tenet of surgical care (2). Skilled resuscitation, known as Battlefield Advanced Trauma Life Support (BATLS) should be delivered within the first hour after wounding, as this is likely to substantially improve survival and long term outcome. Following BATLS, some severe casualties will continue to deteriorate and require surgical intervention to control the immediate life threatening effects of wounding in order to complete their resuscitation. This is known as Damage Control Surgery (3) and should be provided within the second hour for those that require it. Primary surgery describes the first surgery directed at repair of the local damage caused by wounding rather than controlling the immediate life threatening effects. Due to the characteristics of military wounds, further surgical procedures (secondary and tertiary) are normally required, usually in the home base. Primary surgery should be provided within the fourth hour for severe head injuries and no longer than six hours for other injuries.

The collecting zone described by MacPherson (4) usefully describes the e-mail:

mcm.bricknell@btopenworld.com 
collection and sorting of casualties within the forward area. The use of armoured vehicles has added some protection to stretcher bearers. Although these individuals have become considerably better trained in advanced first aid the basic, physically demanding, task of carrying stretchers by hand remains. In the last 20 years battles have been fought in environments that were impassable to vehicles such as mountains (Afghanistan), mud (Falklands), jungles (Panama) and towns (Somalia).

Casualties will be transferred to a vehicle at the earliest point in the evacuation chain. This may be an armoured ambulance in mobile warfare, a helicopter in a jungle clearing or a mule in mountain warfare. This transfer point will be out of small arms fire but is likely to be within range of enemy mortars and artillery. Advanced first aid will be required to treat and package the casualty in order to ensure the best chance for the casualty's survival to the next stage. Triage will be undertaken to put casualties into order for evacuation. This point should also be used as a decision node for the destination of casualties if there is more than one type of medical facility (e.g. a forward surgical hospital for serious cases and a dressing station for less serious casualties). This transfer point is likely to be at the Battalion level and so the convention of having a medical team led by a doctor for this function seems likely to remain.

The next destination for seriously injured casualties should be a surgical hospital. The clinical needs of this group of patients define the capability required from the field medical system as the majority of the technology supporting surgery and postoperative care will meet the needs of all other military patients (e.g. severe chemical casualties or acute medical emergencies). A surgical unit has evolved to meet this task in every war and type of military operation. The previous papers have described Casualty Clearing Stations, Advanced Surgical Centres, Field Surgical Teams, Forward Surgical Teams, Mobile Surgical Hospitals, Hospital Platoons, Mobile Army Surgical Hospitals etc. The common functions and solutions will be discussed later in this paper.

The sick and stable injured form the majority of military patients. The needs of this group define the capacity requirement of the field medical system. Those likely to be returned to duty should be retained as far forward as possible. This almost invariably defines a requirement for a medium and low-dependancy holding facility within the combat area that has been provided by a Dressing Station or Clearing Station. The casualty estimate and the consequent number of beds and medical units supporting the military force will define the linkage between this facility and the surgical and high-dependancy facility. In small operations it may not be necessary to deploy this type of medical unit as the forward surgical hospital can include this capability. When there are large numbers of casualties, the 'triage node' will separate these casualties from the seriously injured patients in the evacuation chain. The specialisation of Casualty Clearing Stations in WW1, the Field Dressing Station of WW2 (British Army) and the modern Combat Support Hospital (US Army) are examples of solutions to this problem.

Nations are increasingly likely to use their national medical systems for the recovery and rehabilitation of their military sick and injured. It is unlikely that any nation will enter a military campaign of such size and duration that specialised units for the longterm convalescence and rehabilitation of military patients will be required in the Theatre of operations. Therefore, a strategic aeromedical evacuation service will be required to transfer patients from the Theatre of operations to the home base. The two 'streams of casualties' (critically ill and the stable) will merge at this stage in the evacuation chain. Patients are likely to be moved in batches through this system and so a form of evacuation hospital is likely to be needed within the logistic support area of the military force. This evacuation hospital should be designed to be deployable to a Theatre of operations and to be re-deployable within the Theatre. More than one evacuation hospital may be needed if there is a long line of communication or if large numbers of casualties are expected. The experience from WW1 and 2 showed benefit in siting hospitals in groups and allocating the receiving hospital task by time periods or by numbers of casualties so as not to overload or exhaust the personnel in these hospitals.

Thus six field medical functions are required to form an evacuation chain: collecting, triage and packaging, forward surgical support, holding and bedding down, evacuation hospital, and casualty transfer (including aeromedical evacuation). The need for intermediate nodes between these functions should be rigorously challenged to ensure that patient care is not compromised. The concept of triage by destination was implicit in Jolly's 'threepoint forward' system, the Hospital Platoons in the US evacuation chain in WW2 and the Hartgill Committee evacuation system. This ensured the earliest possible diversion of seriously injured casualties to a surgical facility away from the flow of the majority of casualties in the evacuation chain. The treatment functions should be joined by a casualty transport system that balances protection, speed, range, cost and simplicity. The combination 
GENERIC MILITARY CASUALTY EVACUATION CHAIN
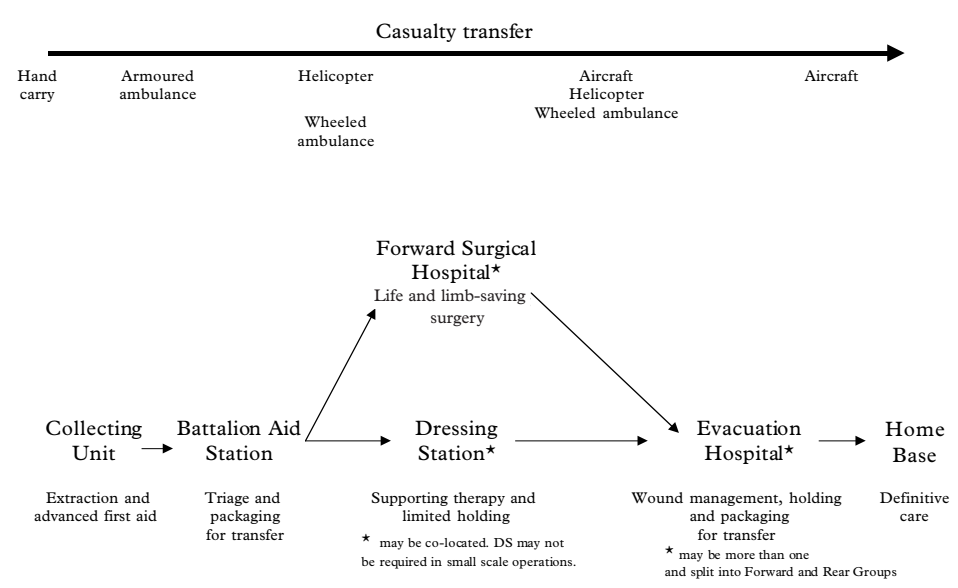

Fig 1. Generic Military Casualty Evacuation Chain.

\begin{tabular}{|c|c|c|}
\hline Topic & Requirement & Remarks \\
\hline $\begin{array}{l}\text { Command and } \\
\text { communications }\end{array}$ & Local commander & $\begin{array}{l}\text { Communication requirement } \\
\text { dependant on local arrangements. } \\
\text { Direct communication between } \\
\text { forward and rear medical facilities } \\
\text { mandatory. }\end{array}$ \\
\hline \multirow[t]{3}{*}{ Mobility } & Overall size & $\begin{array}{l}\text { Basic number of } 25-30 \text { personnel } \\
\text { but increases if } 24 \text { hour nursing } \\
\text { and if the unit is responsible for } \\
\text { its own logistics. }\end{array}$ \\
\hline & $\begin{array}{l}\text { Strategic - } \\
\text { airtransportable }\end{array}$ & $\begin{array}{l}2 \text { C130 loads, dependant on } \\
\text { power, environmental control } \\
\text { and logistic support requirements } \\
\text { and so may deploy in modules. }\end{array}$ \\
\hline & $\begin{array}{l}\text { Tactical - re- } \\
\text { deployable }\end{array}$ & $\begin{array}{l}\text { Integral vehicle lift needed to be } \\
\text { re-deployable in the Theatre of } \\
\text { operations. }\end{array}$ \\
\hline \multirow[t]{7}{*}{ Clinical } & Resuscitation & 2 resuscitation bays. \\
\hline & Primary Care & $\begin{array}{l}\text { Combined with one resuscitation } \\
\text { bay, if an independent clinical } \\
\text { unit. }\end{array}$ \\
\hline & Surgery & $\begin{array}{l}\text { Minimum of general and } \\
\text { orthopaedic surgeon, one table. }\end{array}$ \\
\hline & Intensive Care & $\begin{array}{l}\text { Minimum of two Level } 3 \text { and } \\
\text { two Level } 2 \text { beds per surgical } \\
\text { table. }\end{array}$ \\
\hline & Nursing beds & $\begin{array}{l}\text { Numbers of Level } 1 \text { and Level } 0 \\
\text { bed dependant on time for } \\
\text { evacuation to next treatment } \\
\text { facility. }\end{array}$ \\
\hline & $\mathrm{X}$-ray & Including abdominal ultrasound \\
\hline & Laboratory & $\begin{array}{l}\text { Haematology, biochemistry, } \\
\text { transfusion, microbiology } \\
\text { (dependant on local availability). }\end{array}$ \\
\hline \multirow[t]{4}{*}{ Logistics } & Shelter system & $\begin{array}{l}\text { Weather proof. Heating or air- } \\
\text { conditioning for clinical areas. } \\
\text { Expandable ISO containers if } \\
\text { long deployment to static } \\
\text { location. }\end{array}$ \\
\hline & Electrical supply & Lighting circuit mandatory. \\
\hline & Water supply & Piped water circuit if possible. \\
\hline & $\begin{array}{l}\text { Catering and } \\
\text { accommodation }\end{array}$ & $\begin{array}{l}\text { Dependant on local } \\
\text { arrangements. }\end{array}$ \\
\hline
\end{tabular}

Table 1. Basic Requirements for a Forward Surgical Hospital. of armoured vehicles, wheeled ambulances, helicopters, trains, ships and aeroplanes will be determined by the military campaign. This concept is summarised at Figure 1.

\section{ORGANISATION OF THE FORWARD SURGICAL HOSPITAL}

The forward surgical hospital (FSH) is required to perform surgery on the most seriously injured casualties to 'convert them from may die to will live'. There is some debate whether this should be a discrete clinical unit or whether key components should 'bolt on' to another medical unit. Part 4 of this series described the US Army Mobile Army Surgical Hospital of the Korean War, the modern US Army Forward Surgical Team, the Russian forward surgical unit in Chechnya, the Israeli and Croatian mobile surgical hospitals. These units may work along side sorting and clearing units (e.g. Dressing Stations) but their independence ensures that the mobility of the medical units directly supporting the military formation is retained. The US, Russian and Israeli armies have placed these FSHs under command of the Theatre or Corps medical headquarters for allocation within the combat zone when required. The $\mathrm{FSH}$, its personnel and equipment normally belong to a hospital organisation rather than a divisional medical battalion. This allows the hospital services to develop an incremental, modular concept of operations either by building up from a FSH or drawing down from a full size evacuation hospital. Presently the British philosophy is to augment the Dressing Station for forward surgery but to use hospital troops when post-operative holding is needed. It is possible to establish the basic requirements for a forward surgical unit to support military operations. These are shown in Table 1. Each topic will be discussed in more detail below.

Mission:

To deploy tactically to provide life and limb saving surgery for those casualties who would suffer from delay in further evacuation.

or

To deploy strategically to meet the first mission, provide initial surgery for cases prior to evacuation from theatre, and provide investigation and hospitalisation for disease cases.

\section{COMMAND AND COMMUNICATIONS}

Whilst small, this FSH is a clinically complex organisation that is likely to include senior clinical staff. The work is likely to be emotionally demanding, as a number of patients received by the unit will die. The transfer of casualties from this unit 
is likely to be challenging as they may not be clinically stable and may require sophisticated clinical care in-transit to the next medical unit. An experienced commander should lead the FSH. He should be supported by a communications system that provides a direct link between the FSH, the casualty transfer system and the Evacuation hospital. This is likely to cross the divisional rear boundary and involve the medical staff in the Theatre headquarters. Thus the $\mathrm{FSH}$ requires integral command and communications.

\section{SIZE AND MOBILITY}

The provision of transport to ensure the mobility of the FSH has always been a tension between the medical and logistic staff (1). Parts 1 and 2 of this series described how the British Army was unable to provide integral transport for the light sections of the Casualty Clearing Stations. This resulted in the loss of complete units during the early European campaigns of both WW1 and 2. A forward surgical unit is unlikely to fulfil its' role if it cannot move as an Army advances or withdraws.

The development of air transport enabled military forces to be deployed by air to respond to crises some distance from their base. In addition to tactical mobility on land, the FSH requires strategic mobility by air as described by Shaw in 1969 (5). In 1991, the US Air Force deployed an Airtransportable hospital to Humanitarian Support Base, Silopi, Turkey to support the humanitarian assistance to Kurdish refugees during Operation Provide Comfort (6). Initially a 25-bed facility was set up with 121 personnel. The numbers were reduced to 86 personnel as the Italian, French and British medical facilities became established. In 1999, senior staff from 212th MASH reviewed their unit organisation to create a 'contingency medical forces' module for deployment by air. This unit consisted of 46 personnel and equipment that filled seven air pallets. The unit capability was 4 resuscitation bays, 2 operating tables, 4 Intensive Care beds, 10 medium dependency beds. It was deployed to Albania in support of Task Force HAWK, the US Army component of Joint Task Force Noble Anvil April 2001 (7). The US Air Force has developed an airtransportable medical unit called the Small Portable Expeditionary Aeromedical Rapid Response (SPEARR). This is designed as a modular organisation that has a basic element of 25 personnel to provide a resuscitation department, one operating table and 4 critical care beds. This can be expanded to 10 beds, X-ray and laboratory with 31 extra personnel. An additional 30 personnel increases the capacity to 25 beds. Arising from its deployment to East Timor, the New Zealand Army has also developed an airtransportable hospital unit of 28 personnel. These reports show how a small FSH capability can be used as the basic building block for expansion to increase the capacity of the hospital organisation. The logic for using a hospital organisation as the basis of this function is self-evident.

\section{CLINICAL CAPABILITY}

There is widespread agreement that the FSH capability should include triage and resuscitation, surgery, and post-operative care, supported by laboratory and X-ray (8). Whilst this discussion will concentrate on surgical care, it must be remembered that the Theatre hospital capability must also provide for the management of cases of disease through all ranges of severity. The minimum capability is a resuscitation bay, an operating table (with both a general and orthopaedic surgeon), two intensive care beds and 10-25 nursing beds. These functions will be considered in turn.

\section{The Resuscitation Function}

The previous papers have described the development of resuscitation in military medical units in WW1 and 2. The Field Transfusion Team evolved, with the blood storage and cross-matching function becoming a component of the laboratory and the transfusion officer function becoming incorporated into the resuscitation area of the Dressing Station or field hospital. The Field Surgery Pocket Book of 1962 recommended the appointment of a resuscitation officer to run the resuscitation ward of a forward surgical centre (9). This ward should be capable of holding a large number of casualties and sited close to the operating theatre. Mobile X-ray apparatus, good lighting, pre-laid out trays with standard equipment sets, suction oxygen and cuff sphygmomanometers were regarded as mandatory equipment.

A military medical system of casualty care, Battlefield Trauma Life Support, was introduced into the British Army in 1987. This was a significant step forward in the standardisation of front-line clinical resuscitation. Resuscitation was considered to be the domain of anaesthetists on the basis of their knowledge of physiology. The resuscitation department of 32 Field Hospital in the Gulf war was manned by 4 anaesthetists, 4 general duties medical officers, 8 nursing officers, 8 medical assistants and two bandsmen. This also included the pre-operative holding area. The Gulf War was the first opportunity to evaluate the training protocols from BATLS. This proved its utility in providing a standardised framework for the assessment of a casualty that was understood by all members of the resuscitation team (10).

Accident and Emergency became a formal speciality in the Army in 1994. An Accident 
and Emergency consultant was first deployed on operations to Kosovo in 1999 with 22 Field Hospital (11). This experience led to a review of the organisation and medical equipment of the front end of the field hospital and the formal aggregation of the reception, triage, resuscitation and minor treatment area within the field hospital into the Accident and Emergency Department (12). The minimum capacity for the $\mathrm{A}+\mathrm{E}$ department of a FSH is a single resuscitation bay. Almost invariably a primary care capability will be required and this can form the basis of a second resuscitation bay.

\section{The Surgical Support function}

Surgery. The aim of surgery in a FSH is to control the immediate life threatening effects of wounding in order to complete the resuscitation of a seriously injured casualty. The phase 'damage control surgery' has become synonymous with this capability. However, some surgeons have used the phrase in a more limited context to define abdominal surgery for massive bleeding. In these circumstances, the initial surgical intervention is seen as a temporising measure to halt the physiological deterioration prior to a period of intensive care and planned reoperation when the patient is physiologically stable (13). Other military authors have proposed that the term 'damage control surgery' be extended from a single procedure to that describing 'minimal acceptable care' for circumstances involving large numbers of surgical patients (14). However the clinical definition of 'damage control surgery' has little impact on the process of military medical planning because surgical support will always be required as close as possible to the casualty. The military medical planner has to select the most appropriate capability from available medical units such that the balance of time, quality and capacity are met. The military medical system must ensure that surgical care can be provided after consideration of this balance whatever the definition of damage control surgery.

The military medical histories of WW 1 and 2 include extensive descriptions of the redeployment of Field Surgical Teams both from CCSs forward to ASCs and also between CCSs. Thus a small number of surgical teams (general and orthopaedic) need to have sufficient mobility to be moved between hosting medical units. There is also a clear need for surgical teams (thoracic, head and neck, ophthalmic) that can be attached to evacuation hospitals to provide specialist care for specific groups of patients. This requirement for mobility demands that the procurement of medical and military equipment balances robustness and clinical capability. It is extremely unlikely that all the surgical teams within an evacuation hospital will be re-deployed forward to a FSH or moved between evacuation hospitals. Therefore, it is possible to design different equipment scales for surgical teams that would never re-deploy from a field hospital and for 'field surgical teams' that might be required to re-deploy. This principle may lead to differing scales of equipment that represent a different balance between 'field utility' and 'clinical performance'.

Operational experience in WW 1 and 2, reported in Parts 1 and 2 of this series, showed that multiple operating theatres are best organised within a single shelter. This enables personnel and surgical equipment to be shared between operating tables. 'Nonre-deployable' field hospital surgical teams should be organised as 2 pairs each working from a 18x24 Improved Tented Camp shelter. The shelter could be joined to make an ' $18 \mathrm{x} 48$ ' should four teams be working together. This arrangement allows economy for scarce or expensive items and communal services such as the Central Sterile Supplies Department can be run more efficiently. ISO container-based operating theatres can only hold a single operating team but the clinical environment is usually better than tents (air-conditioning and filtration, water supply etc). These would usually be used in evacuation hospitals where the logistic support for these shelters is more easily available. However these operating shelters might be justified to support small-scale operations of medium duration.

Critical Care. Roberts et al reviewed the provision of intensive care and high dependancy care in the field in 2000 (15). This paper described the provision of critical care services in support of Op AGRICOLA 1 in Macedonia. Critical care is a fundamental component of the acute management of a serious surgical casualty. The organisation of Adult Critical Care Services in the NHS has been reviewed and a definition published (16). There are 4 levels of care:

Level 0 - Patients whose needs can be met through normal ward care in an acute hospital.

Level 1 - Patients at risk of their condition deteriorating, or those recently relocated from higher levels of care, whose needs can be met on an acute ward with additional advice and support from the critical care team.

Level 2 - Patients requiring more detailed observation or intervention including support for a single failing organ system or post-operative care and those 'stepping down' from higher levels of care.

Level 3 - Patients requiring advanced respiratory support alone or basic respiratory support together with support of at least two organ systems. This level includes all complex patients requiring support for multi-organ failure. 
Military critical care services should mirror these developments. It is clear that any operating theatre that is required to treat military trauma cases must be supported by a Level 3 critical care capability if the patient is to be properly managed. This care must be sustained if the casualty is to be moved between facilities to enable forward units to retain mobility. The critical care capability is demanding in terms of personnel and equipment and, therefore, the capacity of the critical care element of a surgical complex should be determined as part of the medical planning cycle. It is suggested that a basic ratio of two Level 3 and two Level 2 beds per operating theatre should be used as an initial planning guide for field hospital surgical teams. Level 1 and Level 0 should be provided on a normal ward of a field hospital and also could be provided within a Dressing Station with appropriate staff. Ex SAIF SEREEA demonstrated that a single $18 \times 24$ ITC tent is suitable for a single critical care area for one surgical team ( 2 Level 3 beds and 2 Level 2 beds). These should be joined if this area is supporting more than one surgical team. The critical care area should be separated into Level 3 and Level 2 shelters if more than two surgical teams are supported. This ensures that the Level 3 area is separated from the turbulence of the Level 2 area, which would also be also being used as a recovery area for the operating theatres.

Sterilising. The phase 'CSSD' is usually used to describe a central sterile supplies department within a fixed hospital environment. In the military environment it tends to be used to describe the sterilising function to support an operating theatre within a clinical facility. These services have been substantially reviewed within the NHS and minimum standards set for the decontamination of medical devices (17). This should represent the standards to be met in the field unless the constraints of the military environment make it impossible (occasionally in a FSH). Each surgical team should be scaled for a sterilising capability. Re-deployable Field Surgical Teams may need individual units. The CSSD for surgical teams permanently attached to evacuation hospitals might share a capability (a true 'central' SSD) as this offers improvements in the decontamination process and equipment savings.

\section{The Clinical Support Function}

For a FSH, Clinical Support encompasses laboratory and imaging. Laboratory services include haematology, biochemistry and transfusion. Microbiology may be required if the FSH is the only deployed hospital capability. The imaging equipment should include ultrasound for the diagnosis of intraabdominal bleeding. X-ray will be required if the FSH is deployed a significant distance from other facilities. An ultrasound-trained radiographer is ideal for substantially enhancing the capability of the imaging facility.

\section{Logistics}

Shelters. The ideal shelter system for the FSH would be simple to establish, weatherproof, robust, provide a suitable clinical environment and easy to operate. No such system currently exists for field use. Tents (soft-walled shelter systems) provide some benefits but are difficult to clean and may not be suitable for the wide range of environmental conditions in which military operations may be conducted (arctic to desert, including chemical and biological environmental contamination). Hard-walled shelter systems have frequently been used to improve on tents. These included the prefabricated huts of WW1 that were replicated almost 80 years later in Vitez in Bosnia by the use of 'Corimec' shelters in 1993 (18). A number of surgical trailers and caravans have been developed during the last century including the Wallace-Cowell theatre trailer in WW1 (19) and a variety of 'lean-tos' and caravans in the desert campaigns of WW2 (20). Caravans offered a better surgical environment but the break-down of the shelter can lead to complete loss of capability. 'Lean to's were adapted to fit the universal 3 ton truck. This meant that the equipment could easily be transferred to another vehicle should the truck become unservicable. In the desert in WW2, the 'lean to' was by far the preferred option for field surgical teams with the caravans being confined to CCSs. The French, German and US military medical services used surgical systems based on expandable ISO containers for the NATO deployment to Bosnia in 1996. In response the British urgently purchased the GIAT system that provided operating theatre and intensive care shelters (21). This system has been deployed to Bosnia, Sierra Leone and Oman. Based on the experience accrued from using the GIAT system, the British Army has let a contract for the delivery of 4 Modular Transportable Surgical Facilities (MTSF) built by Marshall Engineering. There is unlikely to be a single shelter system that meets all the requirements for the FSH and evacuation hospitals. It is important that a range of systems is held in the inventory to enable the best choice to be made for a specific task.

The FSH and the evacuation hospital will need a reliable power circuit for lighting and running electrical equipment. Field expedients for the provision of water need to be developed to provide a better system than pouring water by hand from a jerry can until a piped water supply can be established. A hospital should have heating or air-conditioning to ensure that the temperature of clinical areas (including lab and X-ray) are 
within acceptable limits. Both the FSH and evacuation hospitals need to be able to provide accommodation and feeding for their own personnel and patients, though the size of the FSH can be reduced for a particular task if they are hosted by another unit.

\section{Conclusion}

This series of papers has provided a historical review of the organisation of military casualty evacuation during the 20th century and an evidence-base for future developments. It is proposed that there are six field medical functions required to form an evacuation chain: collecting, triage and packaging, forward surgical support, holding and bedding down, evacuation hospital, and casualty transfer (including aeromedical evacuation). The forward surgical hospital is the key treatment node in the system that will influence clinical outcome. This paper has discussed the Field Surgical Team for such a unit in detail and observed that there is some logic in this team being hosted within an evacuation hospital (to provide flexibility) rather than constraining the specification of the unit by placing it within the establishment of a Role 2 unit.

\section{Acknowledgment}

This project was supported by a short-term research fellowship in the history of medicine from the Wellcome Trust. I am grateful to Captain (Retd) P Starling and the staff of the AMS Museum for their help with the background research for this series of papers.

\section{References}

1. Russell MW. The Role of the Clearing Hospital. f R Army Med Corps 1910;14:603-610.

2. Army Doctrine Publication Volume 3 Logistics Medical Supplement. Ministry of Defence April 2000 .
3. Mattox K. Introduction, background, and future projections of damage control surgery. Surg Clin North Am 1997;77:753-759.

4. MacPherson WG. The removal of the sick and wounded from the battlefield. I R Army Med Corps 1909;12:78-88.

5. Shaw AJ. The medical support of a small air-landed force. F R Army Med Corps 1969;115:19-22.

6. Jennings SA et al. Deployment of an Air Transportable Hospital in Support of Allied Forces during Operation Provide Comfort:April 29 to July 17 1991. Milit Med 1993;158:135-141.

7. Moloff AL and Denny S. The Contingency Medical Force: Chronic Challenge, New Solution. Milit Med. 2001;166:199-203.

8. Facility Design United States Department of Defense NATO Handbook Emergency War Surgery Edition 2: Part III: General Considerations of Wound Management: Chapter XII: Sorting of Casualties http://www.vnh.org/

9. A Field Surgery Pocket Book. HMSO London 1962.

10. Riley B and Mahoney P. Battlefield Trauma Life Support: Its use in the Resusciation Department of 32 Field Hospital during the Gulf War. Milit Med 1996;161;542-546.

11 Hodgetts TJ, Kenward G and Masud S. Lessons form the first operational deployment of emergency medicine. F R Army Med Corps 2000;146:134-42.

12. Bricknell MCM. Organisation and design of Regular field hospitals. F $R$ Army Med Corps 2001;147:161-167.

13. Bowley DMG, Barker P and Boffard KD. Damage control surgery - concepts and practice. F R Army Med Corps 2000;146:176-182.

14. Holcomb JB, Helling TS and Hirshberg A. Military, civilian and rural applications of the damage control philosophy. Milit Med 2001;166:490-493.

15. Roberts MJ, Salmon JB and Salder PJ. The provision of intensive care and high dependancy care in the field. F R Army Med Corps 2000;146:99103.

16. A review of critical care services. Department of Health. www.doh.gov.uk/nhsexec.compcritcare.htm.

17. HSC 1999/179. Controls Assurance in Infection Control. Decontamination of Medical Devices.

18. Army Medical Services Magazine 1993;47:104105.

19. Cowell EM. The Wallace-Cowell Theatre Trailer. $\mathcal{F} R$ Army Med Corps 1916;28:708-722.

20. Crew FAE ed. The Army Medical Services Campaigns Volume II. HMSO London 1957.

21. Army Medical Services Magazine 1996;52:122125. 